Molecules 2010, 15, 3920-3931; doi:10.3390/molecules15063920

Article

\title{
Antifungal Activity and Nail Permeation of Nail Lacquer Containing Piper regnellii (Miq.) C. CD. var. pallescens (C. DC.) Yunck (Piperaceae) Leave Extracts and Derivatives
}

\author{
Andrea Mayumi Koroishi ${ }^{1}$, Elizandra Sehn ${ }^{2}$, Mauro Luciano Baesso ${ }^{2}$, Tânia Ueda-Nakamura ${ }^{1,3}$,
} Celso Vataru Nakamura ${ }^{1,3}$, Diógenes Aparício Garcia Cortez ${ }^{1,4}$ and Benedito Prado Dias Filho ${ }^{1,3} *$

1 Programa de Pós-graduação em Ciências Farmacêuticas, Universidade Estadual de Maringá, Brazil; E-Mail: mayumikoroishi@gmail.com (A.M.K.); tunakamura@uem.br (T.U.N.); cvnakamura@uem.br (C.V.N); dagcortez@uem.br (D.A.G.C.)

2 Pós-graduação em Física, Departamento de Física, Universidade Estadual de Maringá, Brazil; E-Mails: elizandrasehn@hotmail.com (E.S.); mlbaesso@uem.br (M.L.B.)

3 Departamento de Ciências Básicas da Saúde, Universidade Estadual de Maringá, Av. Colombo, 5790, 87020-900, Maringá, Brazil

4 Departamento de Farmácia e Farmacologia, Universidade Estadual de Maringá, Brazil

* Author to whom correspondence should be addressed: bpdfilho@uem.br; Tel.: +55 4430114135 ; Fax: +55 4430114109 .

Received: 31 March 2010; in revised form: 11 May 2010 / Accepted: 12 May 2010 / Published: 1 June 2010

Abstract: The dermatophytes are filamentous fungi that cause cutaneous fungal infections because they use keratin as a nutrient source. For this study the antidermatophyte activity of the extracts and derivates from leaves of Piper regnellii was analyzed. From the dichloromethane extract (EBD) neolignans such as eupomatenoid-3 and eupomatenoid-5 were obtained, and it was submitted to fractionation to remove the green residue, designated as the chloroform fraction (FF). Extracts, chloroform fraction and compounds were tested against Trichophyton rubrum ATCC 28189 to determine the minimum inhibitory concentration (MIC). The chloroform fraction was incorporated to nail lacquer that was analyzed by photoacoustic spectroscopy, in vitro assay and scanning electronic microscopy. For antifungal activity in solid medium the dichloromethane extract and chloroform fraction were used. The compounds eupomatenoid-3 and eupomatenoid-5 were less active than the dichloromethane extract against T. rubrum. EBD and FF showed moderate activity in hyphal growth inhibition in solid medium and EBD did not link to 
ergosterol. Nail lacquer containing the chloroform fraction showed good penetration through the nail as determined by photoacoustic spectroscopy. From in vitro studies it was observed that nail lacquer concentrations above $20 \mathrm{mg} / \mathrm{mL}$ prevented the growth of fungi, but concentrations up to 2.5 inhibited the growth. Scanning electronic microscopy was used to confirm the in vitro nail lacquer activity results. The specie $P$. regnellii showed great antifungal activity against T. rubrum, and nail lacquer containing its chloroform fraction has great potential to treat onychomycosis caused by these microorganisms.

Keywords: Piper regnellii; nail lacquer; dermatophyte; Trichophyton rubrum; onychomycosis

\section{Introduction}

In tropical and subtropical countries, fungal infections are one of the most common skin diseases. Among them, in Brazil, one of the main superficial fungal infections are dermatophytoses. Among the fungal agents responsible for this are the dermatophytes which infect keratinized structures, including nails, hair and skin because they can use the keratin as nutrient source. The genera causing these infections are Epidermophyton, Microsporum and Trichophyton [1]. In the last 30 years, there has been has increased concern about fungal infections due to the increasing the number of immunocompromised patients and individuals who receive chemotherapy treatment like cancer patients and transplant recipients, treated with immunosuppressive drugs [2]. There is a major concern with the effectiveness of antifungals, due to their limitations and also the toxicity of the same, which can lead to the non-recurrence of the fungistatic effect or lead to resistance over the treatment period [3]. Because of this recently chemical and pharmacological studies on substances derived from plants with therapeutic properties are increasingly taking place.

The family Piperaceae, consisting of the genera Piper, Peperomia, Ottonia and Pothomorphe, has medicinal properties widely employed by the population [4]. Regarding the genus Piper, phytochemical searches worldwide have isolated many classes of bioactive compounds like alkaloids, amides, propenylphenols, lignans, neolignans, terpens, chalcones, flavones and many others [5]. This genus displays a variety of biological activities, for example the antifungal activity of Piper arboreum and Piper tuberculatum against Cladosporium cladosporioides [6], Piper fulvescens against Trichophyton mentagrophytes and Microsporum gypseum [7,8], moreover, the specie Piper regnellii showed activity against Staphylococcus aureus and Bacillus subtilis, yeasts Candida krusei and Candida albicans [9], dermtophyte Trichophyton rubrum, T. mentagrophytes, Microsporum canis, M. gypseum [10], protozoa like Leishmania amazonensis [11] and Trypanossoma cruzi [12].

The human nail is composed by highly compressed and keratinized dead cells. Many nail disorders may occur such as dystrophy, hypertrophy, inflammation, infection, etc [13]. Onychomycosis is a type of ungual disorder. The main fungi that cause this pathology are Epidermophyton floccosum, $T$. mentagrophytes and T. rubrum [14].

Oral therapy displays some disadvantages such as systemic adverse effects and drug interactions. To work around this, topical therapy is advantageous because the drug acts directly at the site of action 
and this possibly reduces the side effects, drug interactions and costs of treatment [15]. The use of nail lacquer formulations containing an antimycotic may act as a regular transungual delivery system thereby facilitating the penetration of the active principles into the nail $[15,16]$.

The aim of this study was to determine the antifungal activity against $T$. rubrum of extract, fractions and compounds from leaves of $P$. regnellii. In addition, a nail lacquer containing the chloroform fraction to treat onychomycosis was developed and the activity of this formulation was analyzed by photoacoustic spectroscopy, in vitro assay and scanning electronic microscopy.

\section{Results and Discussion}

A 9:1 hydroethanolic extract, a dichloromethane extract (EBD) and an aqueous extract were obtained from the leaves of plant $P$. regnelli. The EBD was clarified using chloroform as mobile phase, and then called the chloroform fraction (FF). In addition, compounds as eupomatenoid-3 and eupomatenoid-5 were isolated from the EBD. All the extracts and derivates were submitted to the antifungal activity assay.

The minimal inhibitory concentration of the extract was $15.6 \mu \mathrm{g} / \mathrm{mL}$, as described by Koroishi et al. [10] and that of the chloroform fraction was $7.8 \mu \mathrm{g} / \mathrm{mL}$. The two isolated neolignans, eupomatenoid-3 and eupomatenoid-5; showed activity greater than $100 \mu \mathrm{g} / \mathrm{mL}$ and $25 \mu \mathrm{g} / \mathrm{mL}$, respectively. Nystatin, ketoconazole, fluconazole and amphotericin B were used as standard drugs, and their MIC values were $0.78,0.39,3.2$ and $0.39 \mu \mathrm{g} / \mathrm{mL}$, respectively (Table 1 ). The minimal concentration values required for inhibition of spore germination are also presented in Table 1 . The results were satisfactory. The MIC values of the extract and fractions were not significantly different, showing that even after removal of the dark green residue using chloroform in the clarification process the activity is retained. In previous studies different hydroethanolic extracts of the same plant were tested against yeasts, dermatophytes and non-dermatophytes, and the microorganisms most sensitive to the extracts were dermatophytes, $T$. rubrum, T. mentagrophytes, M. canis and M. gypseum [10]. Other Piper species contain compounds with antifungal activity, such as the amides of Piper arboreum and Piper tuberculatum, active against Cladosporium sphaerospermum and C. cladosporioides, respectively [6]. Danelutte [17] examined the activity of flavonones and prenylated hydroquinones of Piper crassinervium against the same fungi, which varied between 1 and 5, and 1 to $10 \mu \mathrm{g} / \mathrm{mL}$, Freixa et al [8] studied the activity of three neolignans of Piper fulvescens, eupomatenoid-5, eupomatenoid-6 and conocarpan, and only the first one was not active against the dermatophyte $T$. mentagrophytes, while the other compounds showed activity at 1 and $8 \mu \mathrm{g} / \mathrm{mL}$, respectively. Many other species have been studied for antidermatophyte activity, like species of the family Asteraceae,. For example, the chloroform extract of Pterocaulon polystachyum showed better activity at a concentration of $12.5 \mu \mathrm{g} / \mathrm{mL}$ against $T$. mentagrophytes [2]. Prasad et al. [18] checked the activity of 4'-methoxyflavone, a compound isolated of Psoralea coryfolia (Fabaceae), with a MIC value of $62.5 \mu \mathrm{g} / \mathrm{mL}$ vs. T. mentagrophytes and T. rubrum and 125 $\mu \mathrm{g} / \mathrm{mL}$ against E. floccosum and M. gypseum. Among other species Ziziphus joazeiro Mart. (Rhamanaceae) and Caesalpinia pyramidalis Tul are active against Candida guilliermondii and $T$. rubrum with MIC values between 6.25 and $25 \mu \mathrm{g} / \mathrm{mL}$. In previous studies it was verified that the extracts obtained of leaves from $P$. regnellii significantly inhibited the germination of $T$. rubrum spores at concentration of 7.8 . 
Table 1. Antifungal activity of extract, fraction and isolated compounds of leaves from $P$. regnellii against $T$. rubrum.

\begin{tabular}{lcccc}
\hline & \multicolumn{3}{c}{ Antifungal Activity } \\
\cline { 2 - 4 } & $\begin{array}{c}\text { Minimal } \\
\text { required } \\
\text { spore germination }(\boldsymbol{\mu g} / \mathbf{m L})\end{array}$ & $\begin{array}{c}\text { concentration } \\
\text { inhibition of }\end{array}$ & $\begin{array}{c}\text { Minimal } \\
\text { concentration }(\boldsymbol{\mu g} / \mathbf{m L})\end{array}$ \\
\hline Dichloromethane extract (EBD) & 7.8 & 15.6 \\
Chloroform fraction (FF) & 7.8 & 7.8 \\
Eupomatenoid-3 & $>100$ & $>100$ \\
Eupomatenoid-5 & 12.5 & 25 \\
Amphotericin B & 0.2 & 0.4 \\
Nystatin & 0.4 & 0.8 \\
Fluconazole & 1.6 & 3.2 \\
Ketoconazole & 0.2 & 0.4 \\
\hline
\end{tabular}

There are two phases of fungal growth, spore germination and hyphal growth, where drug action can occur. The hyphal growth inhibition was analyzed and the extract and fraction showed moderate inhibition at a concentration of $100 \mu \mathrm{g} / \mathrm{mL}$, while amphotericin B inhibited it at $10 \mu \mathrm{g} / \mathrm{mL}$, as observed in the Figure 1. DMSO and water were employed as controls.

Figure 1. Antifungal active in solid medium against T. rubrum. $\mathrm{A}=$ Negative control DMSO 100, 10, 1, 0.1\%; B= Amphotericin B - 100, 10, 1, $0.1 \mu \mathrm{g} / \mathrm{mL}$ and water. $\mathrm{C}=$ Dichloromethane extract $(\mathrm{EBD})$ and $\mathrm{D}=$ Chloroform fraction $(\mathrm{FF})-1000,100,10$ and $1 \mu \mathrm{g} / \mathrm{mL}$. The water was used as control, and the concentrations decrease in clockwise fashion.
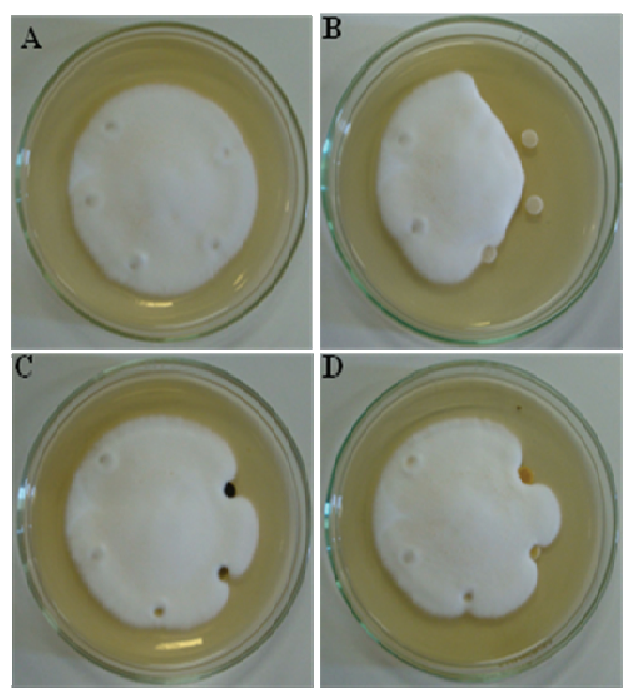

Many different modes of action can be studied, for example, the biosynthesis of ergosterol or the interference of drug-ergosterol binding with cell functions. Ergosterol is lipid that forms part of the membranes of fungi and protozoa. For example amphotericin B binds to ergosterol perturbing membrane functions and causing leakage of cell contents [19]. Figure 2 shows the effect of exogenous ergosterol on the MIC of the drugs. 
Figure 2. Ergosterol effect assay in T. rubrum - Exogen ergoterol $(62.5-500 \mu \mathrm{g} / \mathrm{mL})$ was added on the MIC of the dichlomethane extract (EBD), amphotericin B (Amp B) and nystatin (Nys).

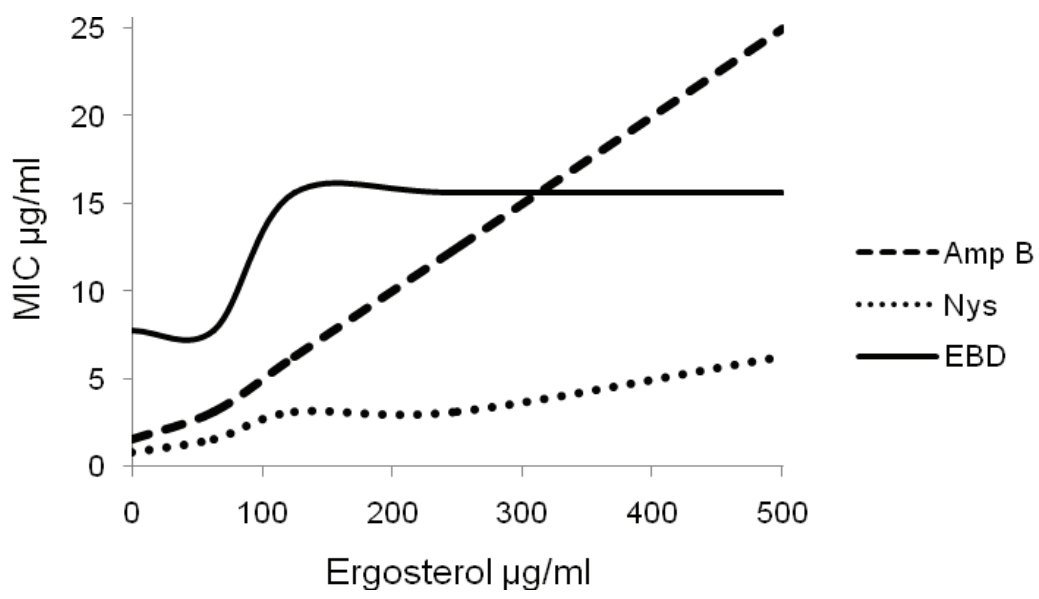

When the concentrations of ergosterol increase the values of the MIC of amphotericin B also increase. In the absence of ergosterol the MIC of this drug was $1.56 \mu \mathrm{g} / \mathrm{mL}$, and by varying the substrate between 0 to $500 \mu \mathrm{g} / \mathrm{mL}$, the MIC changed from 1.56 to $25 \mu \mathrm{g} / \mathrm{mL}$, indicating binding with the ergosterol. Despite this, the extract and nystatin did not have the same activity as amphotericin B, because when the ergosterol concentration increased the MIC values remained the same.

Figure 3. Antifungal activity of nail lacquer with different concentrations of extract against T. rubrum. $\mathrm{A}=$ Control; $\mathrm{B}=$ Nail lacquer without fraction; Nail lacquer with fraction: $\mathrm{C}=2.5 \mathrm{mg} / \mathrm{mL} ; \mathrm{D}=5 \mathrm{mg} / \mathrm{mL} ; \mathrm{E}=10 \mathrm{mg} / \mathrm{mL} ; \mathrm{F}=20 \mathrm{mg} / \mathrm{mL} ; \mathrm{G}=40 \mathrm{mg} / \mathrm{mL} ; \mathrm{H}=$ Micolamin.

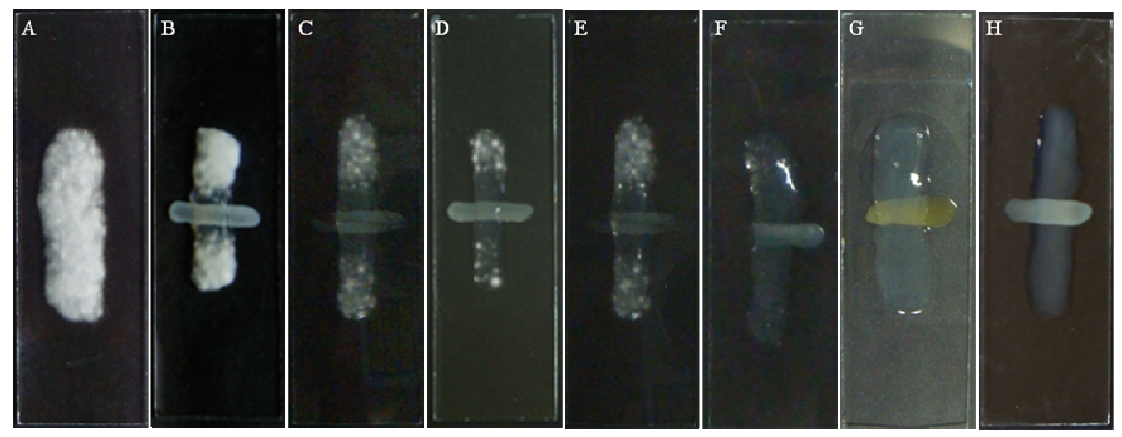

The chloroform fraction was incorporated into nail lacquer at various concentrations ranging between 0.3 to $40 \mathrm{mg}$ per $\mathrm{mL}$. The concentration of $20 \mathrm{mg} / \mathrm{mL}$ did not present visible growth compared the control (Figures $3 \mathrm{~F}$ and $3 \mathrm{~A}$ ). However, concentrations above $2.5 \mathrm{mg} / \mathrm{mL}$ inhibited the growth of fungi (Figure 3C) at the point of application of the nail lacquer. Figure 4 shows images of MEV of T. rubrum. As previously mentioned, in this method was possible to confirm the reduction of the growth of cultures treated with nail lacquer containing chloroform fraction. There is a reduced growth of cells treated near the nail lacquer (Figures 4C, 4E and 4F). Comparing the figures 4D, 4F and $4 \mathrm{G}$ could see cell growth was lower at a concentration of $5 \mathrm{mg} / \mathrm{mL}$. It is possible the active compounds spread by the agar and in higher concentrations the compounds inhibited the growth. 
Figure 4. Scanning electronic microscopy of Trichophyton rubrum treated with different concentrations of fraction in nail laquer. $\mathrm{A}$ and $\mathrm{B}=$ control; $\mathrm{C}$ and $\mathrm{D}=$ nail lacquer without fraction; $\mathrm{E}$ and $\mathrm{F}=$ nail lacquer with $1.25 \mathrm{mg}$ of chloroform fraction; $\mathrm{G}$ and $\mathrm{H}=$ nail lacquer with $5 \mathrm{mg}$ of chloroform fraction. $\mathrm{C}, \mathrm{E}$ and $\mathrm{G}=$ samples taken near the nail lacquer; $\mathrm{D}, \mathrm{F}$ and $\mathrm{H}=$ samples taken far the nail lacquer. Magnification: $3000 \times(\mathrm{A})$ and $2000 \times(\mathrm{B}-\mathrm{H})$. Bars $5 \mu \mathrm{m}$.

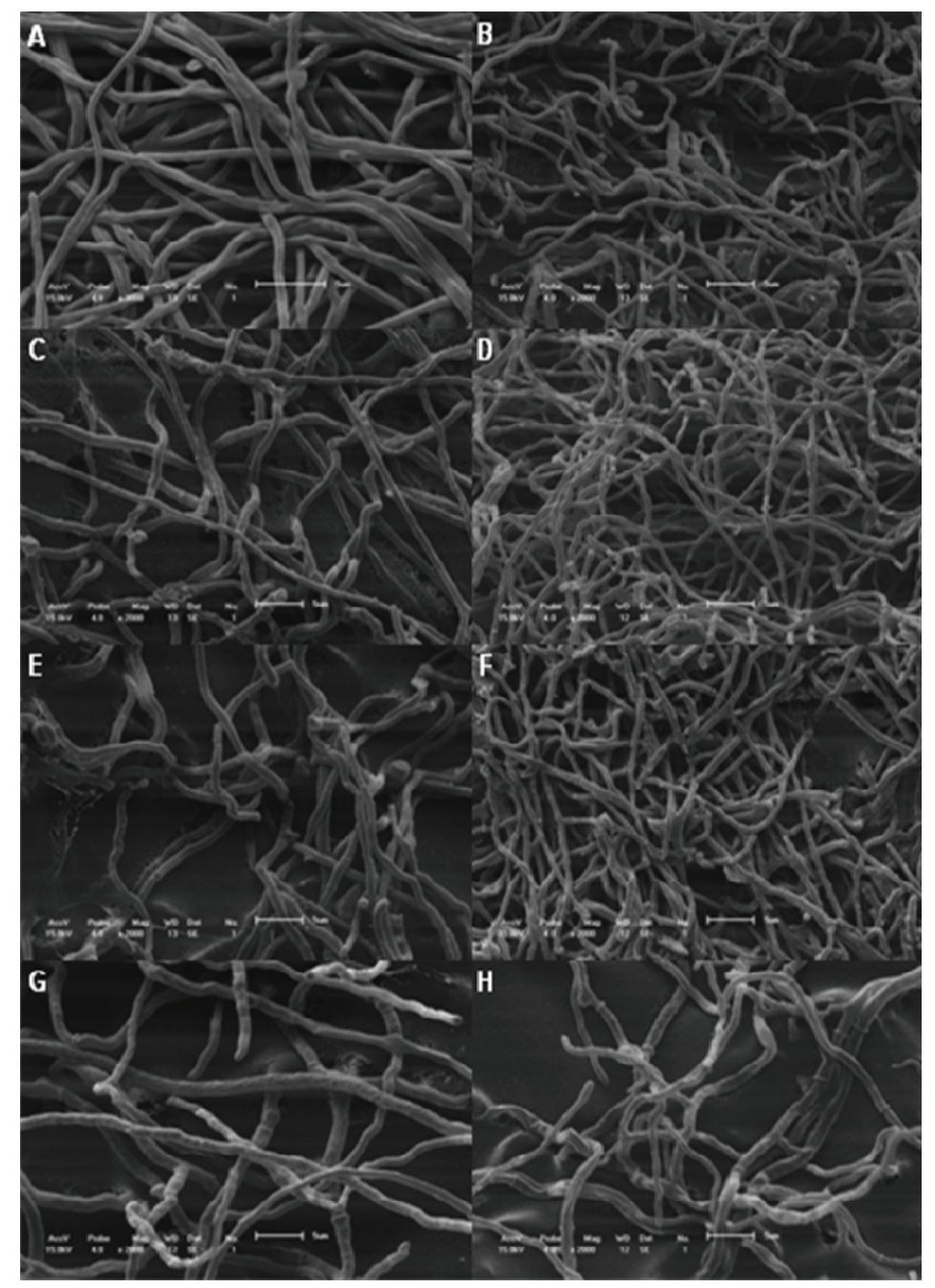

The human nail is susceptible to a large number of fungal (onychomycosis) and other infections [21]. The photoacoustic spectroscopy technique if used to measure the thermal diffusivity $(\alpha)$ of human nails [21]. Figure 5 shows the spectra of the drugs on the nail. The medicine was placed on top of the nail and a top spectrum was obtained soon after. The graphic shows the curve of the absorption spectrum of the bottom of the nail $30 \mathrm{~min}$ after application of FF fraction. After $5 \mathrm{~h}$ of application of the product, could be performing the spectrum observed in the inner part of the permeation of the nail. The band around $670 \mathrm{~nm}$ becomes visible, showing the permeation. The nail lacquer containing the FF fraction showed good penetration in the nail and the absorption is still equal after extending the time. Nuglisch et al [22] verified that using two formulations contained cyclopirox the propagation rate through the nails in different samples from healthy volunteers showed that the photoacoustic spectroscopy technique is a useful tool to analyze the penetration of topical formulations in human nails. 
Figure 5. Photoacoustic Spectroscopy Measurement. after 20 min the FF fraction was detected at $670 \mathrm{~nm}$. Spectra obtained with excitation performed in the nail. The light modulation frequency was $20 \mathrm{~Hz}$. The inset highlights the presence of the FF fraction absorption in the spectrum. With this procedure the penetration depth in the nail (the thermal diffusion length) corresponds to about $670 \mathrm{~nm}$.

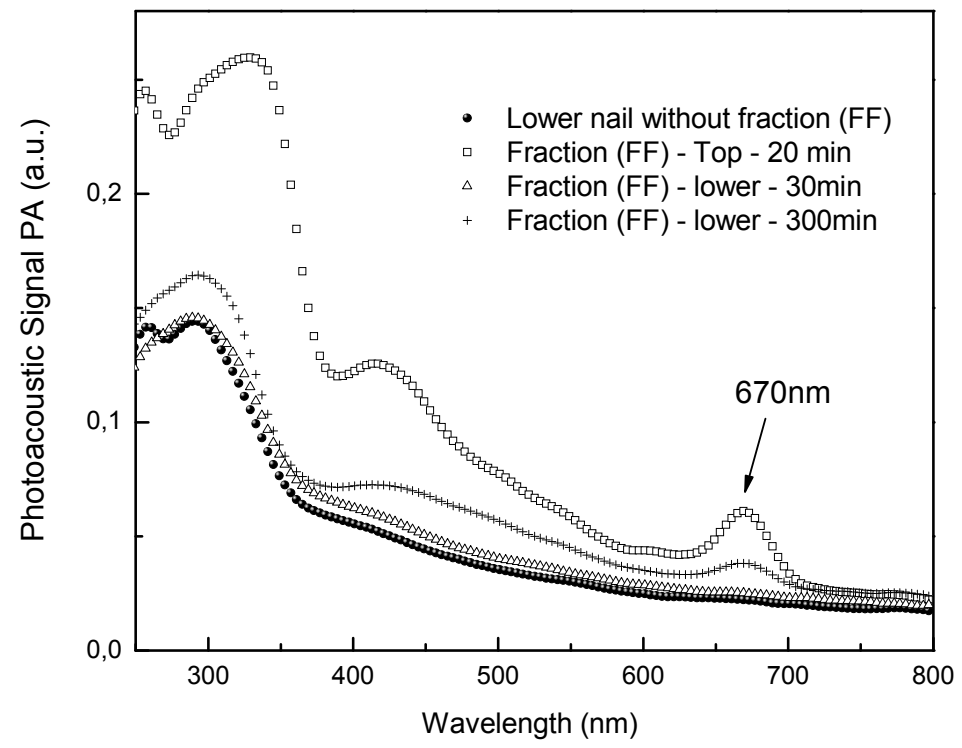

\section{Experimental}

\subsection{Plant material}

The leaves from Piper regnellii (Miq.) C. CD. var. pallescens (C. DC.) Yunck (Piperaceae) were collected in April 2006 in Horto of Medicinal Plants "Profa. Irenice Silva" on the Campus of Universidade Estadual de Maringá. The plant material was identified by Marilia Borgo of the Botanical Department of Universidade Federal do Paraná, and a voucher specimen (HUM 8392) is deposited at the Herbarium of Universidade Estadual de Maringá, Paraná, Brazil.

\subsection{Preparation of plant extract and fractions}

Dried and powdered plant material (leaves) was extracted by maceration with 9:1 ethanol-water at room temperature at a leaves:solvent ratio of 1:10 (w/v). After the extract was filtered and the filtrate was evaporated to dryness under reduced pressure at $40{ }^{\circ} \mathrm{C}$ to give an aqueous extract and a dark green residue which was washed with dicloromethane and the organic solvent removed to give the dicloromethane extract that was submitted to two processes. First, to isolate the compounds eupomatenoid-3 and eupomatenoid-5 the techniques already described in previous studies [10,12,20] were used. The extract was submitted to vacuum chromatography on silica gel eluted with solvents of increasing polarity. The hexane fraction, obtained by this process, was rechromatographed by column chromatography on silica gel 60 (70-230 mesh) eluted with hexane and hexane-chloroform in the following proportions: 49:1, 19:1, 9:1 and 1:1, v/v, chloroform, ethyl acetate, acetone and methanol. 
The second process, to remove (clarify) the green residue was used $D$-chloroform colunm eluated with chloroform, obtaining the chloroform fraction.

\subsection{Nail lacquer formulation}

The formulation used consisted of a solvent volatile (ethanol, ethyl acetate, methanol), apolar soluble polymer (methacrylic acid copolymer, vinyl polymers), plasticizer (triacetine, dibutyl vitalato) and the chloroform fraction, at concentrations between 0.6 to $40 \mathrm{mg} / \mathrm{mL}$.

\subsection{Microorganism used and growth conditions}

The test specie used for this investigation was T. rubrun ATCC 28189. The fungi was maintained on Sabouraud dextrose agar (SDA) slants at $28{ }^{\circ} \mathrm{C}$ and subcultured monthly throughout this study.

\subsection{Antifungal activity assay}

\subsubsection{Microbroth dilution assay}

Culture was grown on Sabouraud dextrose agar (SDA, Difco Laboratories, Detroit, MI, USA) tubes for 7-14 days, after which time spores were harvested from sporulating colonies and suspended in sterile ion solution. The concentration of spores was adjusted to $1.0 \times 10^{5}$ spores $/ \mathrm{mL}$ using a hemocytometer. The antifungal assay was performed by the microdilution technique in sterile flat botton microplates. Each well contained appropriate test samples, Sabouraud dextrose broth and approximately $2 \times 10^{3}-3 \times 10^{3}$ spores in a total volume of $100 \mu \mathrm{L}$. The plates were incubated at $28^{\circ} \mathrm{C}$ for $72 \mathrm{~h}$. Two susceptibility endpoints were recorded for each isolate. The MIC was defined as the lowest concentration of compounds at which the microorganism tested did not demonstrate visible growth. For this experiment the dichloromethane extract, the chloroform fraction, the isolated compounds and nystatin, amphotericin, fluconazole and ketoconazole at concentrations between 0.2 to $100 \mu \mathrm{g} / \mathrm{mL}$ as standard drugs were used. For comparative purposes, the plates were incubated at $28{ }^{\circ} \mathrm{C}$ for 20-30 $\mathrm{h}$ and then examined for spore germination under an inverted microscope. For quantification, spores were considered germinated if they had a germ tube at least twice the length of the spore.

\subsubsection{Antifungal activity in solid medium}

Petri-plates containing $20 \mathrm{~mL}$ de SDA medium were seeded with $10 \mu \mathrm{L}$ of standard spore suspension $\left(10^{5}\right.$ spores $\left./ \mathrm{mL}\right)$. The plates were incubated in a humidified chamber at a temperature of $28{ }^{\circ} \mathrm{C}$ until the diameter growth mycelial growth $2-3 \mathrm{~cm}$. After, the disks were placed at a distance of $0.5 \mathrm{~cm}$ from the edge of the colony. About $10 \mu \mathrm{L}$ of serial dilutions of dichloromethane extract, chloroform fraction, DMSO, and water were added to the disks. The plates were incubated at a temperature of $28{ }^{\circ} \mathrm{C}$ until final growth. The inhibition of hyphal elongation was observed by the lack of growth around the disk containing the drug test [18] 


\subsubsection{Ergosterol Effect Assay}

For this assay, the ergosterol was added the Sabouraud dextrose broth medium at four concentrations: $500,250,125$ and $62.5 \mu \mathrm{g} / \mathrm{mL}$. The dichloromethane extract was transferred to the first well, a two fold serial dilution was perfomed and the medium was incubated at $28{ }^{\circ} \mathrm{C}$ for $72 \mathrm{~h}$. Amphotericin B and nystatin were used as controls [3].

\subsubsection{In vitro test with the nail lacquer containing fraction}

In this test, the nail lacquer was applied at glass slides forming a line. Concentrations between 0.3 to $40 \mathrm{mg} / \mathrm{mL}$ were analyzed, and Micolamin was used as control drug. The inoculum used had $3 \times 10^{4}$ spores $/ \mathrm{mL}$. The microculture was placed in a Petri dish in a moist chamber at a temperature of $28{ }^{\circ} \mathrm{C}$ for 5 days. Later, the cultures underwent scanning electronic microscopy. Three portions were removed, far, near and upon the application point.

\subsubsection{Scanning electronic microscopy}

The microculture was fixed in glutaraldehyde $2.5 \%$ in $0.1 \mathrm{M}$ cacodilato buffer for 1 day. Later, it was washed in $0.1 \mathrm{M}$ cacodilato buffer. The samples were placed on a specimen support with poly-Llysine. Subsequently, the samples were dehydrated in graded ethanol, 15-100\% for 15 min each. Then, they were submitted to critical-point-drying in $\mathrm{CO}_{2}$, coated with chromium in a Penning sputter system in a high-vaccum chamber chamber (Gatan-Model 681), coated with gold and observed with a Shimadzu SS-550 Scanning electron microscope field-emission scanning electron microscope.

\subsection{Photoacoustic Spectroscopy Measurements}

The photoacoustic spectroscopy (PAS) measurements were performed using an experimental setup as shown in Figure 5. The monochromatic light was obtained from a $1000 \mathrm{~W}$ xenon arc lamp (Oriel Corporation 68820). The monochromator used was also from Oriel Instruments (model 77250). The light beam was modulated with a mechanical chopper (Stanford Research Systems SR540). The photoacoustic cell was homemade projected to have a minimal volume. It was made of aluminum block, machined to hold samples with maximum dimensions of about $5 \mathrm{~mm}$ in diameter and $1 \mathrm{~mm}$ thick, which allows light to enter through a high transparent quartz window of $6 \mathrm{~mm}$ in diameter and $2 \mathrm{~mm}$ thick. The micro-phone chamber was $15 \mathrm{~mm}$ away and connected to the sample holder chamber by means of a $1 \mathrm{~mm}$ diameter duct. The used capacitive microphone is a very sensitive $12 \mathrm{~mm}$ diameter Bruel \& Kjaer model 2639, which presents a high gain of $50 \mathrm{mV} / \mathrm{Pa}$ and flat frequency response performance from $1 \mathrm{~Hz}$ to $10 \mathrm{kHz}$. The lock-in amplifier was from EG \& $\mathrm{G}$ Instruments, model 5110. All the photoacoustic spectra were obtained at a modulation frequency of $25 \mathrm{~Hz}$ and recorded between 250 and $800 \mathrm{~nm}$. The data acquisition was performed by a personal computer and the PAS spectra were normalized with respect to the carbon black signal. In the photoacoustic measurements, the thermal diffusion length $\left(\mu_{\mathrm{s}}\right)$ defines the sample nail [22] depth which contributes to 
the signal. This parameter is defined as $\mu_{\mathrm{s}}=(\mathrm{d} / \alpha \mathrm{f})^{1 / 2}$, in which $\mathrm{d}$ is the sample thermal diffusivity and $\mathrm{f}$ the light modulation frequency. With low frequencies one can inspect at long depths beneath the nail surface, while higher frequencies probe the nail surface. This is the well known characteristic of this technique, widely used to perform depth profile analysis. Taking $\mathrm{f}=25 \mathrm{~Hz}$ and the thermal diffusivity of the nail measured before as $\mathrm{d}=8.1 \times 10^{-4} \mathrm{~cm}^{2} / \mathrm{s}$ [23], the studied samples present thicknesses varying from 400 to $600 \mu \mathrm{m}$. The samples were excited first onto the sample external face and after that they were turned upside down to impinge the light in the sample internal face. In this way, the detection of the fraction FF optical absorption bands at the internal side of the sample means that the applied substances propagated through the wound.

\section{Conclusions}

In conclusion, Piper regnellii showed effective antifungal activity, although the isolated compounds eupomatenoid-3 and eupomatenoid-5 showed low activity when compared the dichloromethane extract and chloroform fraction. The incorporation of this fraction in a vehicle, nail lacquer, showed good permeation through the nail and good in vitro activity against T. rubrum. Furthermore, the dichloromethane extract was not able to bind ergosterol.

\section{Acknowledgements}

Programa de Pós-graduação em Ciências Farmacêuticas da Universidade Estadual de Maringá, CAPES/CNPq, Fundação Araucária.

\section{References and Notes}

1. Weitzman, I.; Summerbell, R.C. The dermatophytes. Clin. Microb. Rev. 1995, 8, 240-259.

2. Stein, A.C.; Sortino, M.; Avancini, C.; Zacchino, S.; Poser, G. Ethnoveterinary medicine in the search for antimicrobial agents: Antifungal activity of some species of Pterocaulon (Asteraceae). J. Ethnopharmacol. 2005, 99, 211-214.

3. Sortino, M.; Delgado, P.; Juárez, S.; Quiroga, J.; Abonía, R.; Insuasty, B.; Nogueras, M.; Rodero, L.; Garibotto, F.M.; Enriz, R.D.; Zacchino, S.A. Synthesis and antifungal activity of (Z)-5aryldenerhodanines. Bioorg. Med. Chem. 2007, 15, 484-494.

4. Costa, A.F. Farmacognosia (Farmacognosia Experimental); Fundação Calouste Gulbenkian: Lisboa, Portugal, 1972; p. 380.

5. Parmar, V.S.; Jain, S.C.; Bisht, K.S.; Jain, R.; Taneja, P.; Jha, A.; Tyagi, O.D.; Prasad, A.K.; Wengel, J.; Olsen, C.E.; Boll, P.M. Phytochemistry of the genus Piper. Phytochemistry 1997, 6, 597-673.

6. Silva, R.V.; Navickiene, H.M.D.; Kato, M.J.; Bolzani, V.S.; Méda, C.I.; Young, M.C.M.; Furlan, M. Antifungal amides from Piper arboreum and Piper tuberculatum. Phytochemistry 2002, 59, 521-527.

7. Freixa, B.; Vila, R.; Vargas, L.; Lozano, N.; Adzet, T.; Cañigueral, S. Screening for antifungal activity of nineteen Latin American plants. Phytother. Res. 1998, 12, 427-430. 
8. Freixa, B.; Vila, R.; Ferro, E.A.; Adzet, T.; Cañigueral, S. Antifungal principles from Piper fulvescens. Planta Med. 2001, 67, 873-875.

9. Holetz, F.B.; Pessini, G.L.; Sanches, N.R.; Cortez, D.A.G.; Nakamura, C.V.; Dias Filho, B.P. Screening of some plants used in brazilian folk medicine for the treatment of infectious diseases. Mem. Inst. Oswaldo Cruz 2002, 97, 1027-1031.

10. Koroishi, A.M.; Foss, S.R.; Cortez. D.A.G.; Ueda-Nakamura, T.; Nakamura, C.V.; Dias Filho, B.P. In vitro activity of extracts and neolignans from Piper regnellii against dermatophytes. $J$. Ethnopharmacol. 2008, 117, 270-277.

11. Vendrametto, M.C.; Santos, A.O.; Nakamura, C.V.; Dias Filho, B.P.; Cortez, D.A.G.; UedaNakamura, T. Evaluation of antileishmanial activity of eupomatenoid-5, a compound isolated from leaves of Piper regnellii var. pallescens. Parasitol. Int. 2010, 59, 154-158.

12. Luize, P.S.; Ueda-nakamura, T.; Dias Filho, B.P.; Cortez, D.A.G.; Nakamura, C.V. Activity of neolignans isolated from Piper regnellii (Miq.) C. DC. var. pallescens (C. DC.) YUNCK against Trypanosoma cruzi. Biol. Pharm. Bull. 2006, 29, 2126-2130.

13. Murdan, S. Drug delivery to the nail following topical application. Int. J. Pharm. 2002, 236, 1-26.

14. Elewski, B. Onychomycosis: pathogenesis, diagnosis and management. Clinical Microb. Rev. 1998, 11, 415-429.

15. Effendy, I. Therapeutic strategies in onychomycosis. J. Eur. Acad. Derm. Venereol. 1995, 4, S3-S10.

16. Marty, Jean-paul, L. Amorolfine nail lacquer: a novel formulation. J. Eur. Acad. Derm. Venereol. 1995, 4, S17-S21.

17. Danelutte, A.P.; Lago, J.H.; Young, M.C.M.; Kato, M.J. Antifungal flavanones and prenylated hydroquinones from Piper crassinervium Kunth. Phytochemistry 2003, 64, 555-559.

18. Prasad, N.R.; Anandi, C.; Balasubramanian, S.; Pugalendi, K.V. Antidermatophyte activity of extracts from Psoralea corylifolia (Fabaceae) correlated with the presence of a flavonoid compound. J. Ethnopharmacol. 2001, 91, 21-24.

19. Odds, F.C.; Brown, A.J.P.; Gow, N.A.R. Antifungal agents: mechanisms of action. Trends Microbiol. 2003, 11, 272-279.

20. Pessini, G.L.; Dias Filho, B.P.; Nakamura, C.V.; Cortez, D.A.G. Antibacterial activity of extracts and neolignans from Piper regnellii (Miq.) C. DC. var pallescens (C. DC.) Yunck. Mem. Inst. Oswaldo Cruz 2003, 98, 1115-1120.

21. Sowa, M.G.; Wang, J.; Schultz, C.P.; Ahmed, K.; Mantsch, H.H. Infrared spectroscopy investigation of in vivo and ex vivo human nails. Vib. Spectrosc. 1995, 10, 49-56.

22. Nuglisch, L.E.R.; Dias, D.T.; Sehn, E.; Bento, A.C.; Baesso, M.L.; Santos, S.T.S.; Fushimi, M.Y. Photoacoustic spectroscopy to evaluate the penetration of two antifungal agents through the human nail. J. Phys. IV 2005, 125, 631-633.

23. Dias, D.T.; Nuglish, L.E.R.; Sehn, E.; Baesso, M.L.; Medina, A.N.; Bento, A.C. Human nail thermal diffusivity obtained using the open photoacoustic cell technique. J. Phys. IV 2005, 125, 657-660. 
Sample Availability: Samples of the compounds are available from the authors.

(C) 2010 by the authors; licensee MDPI, Basel, Switzerland. This article is an Open Access article distributed under the terms and conditions of the Creative Commons Attribution license (http://creativecommons.org/licenses/by/3.0/). 